\title{
Playing to our human strengths to prepare medical students for the future
}

\section{Julie Chen}

Department of Family Medicine and Primary Care, Bau Institute of Medical and Health Sciences Education, The University of Hong Kong, Hong Kong

We are living in an age where artificial intelligence and astounding technological advances are bringing truly remarkable change to healthcare. Medical knowledge and skills which form the core responsibility of doctors such as making diagnoses may increasingly be delivered by robots. Machines are gradually acquiring human abilities such as deep learning and empathy. What, then is the role of doctors in future healthcare? And what direction should medical schools be taking to prepare their graduates? This article will give an overview of the evolving technological landscape of healthcare and examine the issues undergraduate medical education may have to address. The experience at The University of Hong Kong will serve as a case study featuring several curricular innovations that aim to empower medical graduates with the capabilities to thrive in the future.

Key Words: Curriculum, Undergraduate, Technology, Competency, Hong Kong

\section{Introduction}

Undergraduate medical education has been under scrutiny at critical junctures in history. Each time, medical education has responded. Flexner's 1910 report established the biomedical model and university-based training as the standard for medical training, and more recently the 2010 Lancet commissioned report on medical education argued for curricula that emphasize professionalism, social responsibility, and inter-professional and competency-based standards [1]. In this era of rapidly advancing technology and artificial intelligence, medical education is at another critical juncture. It is now facing the double-edged sword of the challenges and promises brought forth by a changing technologicallyrich environment of practice. This raises significant questions for medical educators to consider going forward: (1) What is medical practice going to look like in the coming decades? (2) Who are the medical students who will be the doctors practising medicine in the future? (3) How can medical education address both issues?

\section{Medical practice: a brave new world}

Knowledge and skills are two of the fundamental
Received: July 31, 2017 • Revised: July 31, 2017 • Accepted: July 31, 2017 Corresponding Author: Julie Chen (https://orcid.org/0000-0002-7444-6182) Department of Family Medicine and Primary Care, The University of Hong Kong, Room A5-10, 5/F William MW Mong Block, 21 Sassoon Road, Pokfulam, Hong Kong Tel: +852.2518.5657 Fax: +852.2814.7475 email: juliechen@hku.hk

* This article is based on a symposium talk given on June 2, 2017 at the 33rd Korean Society of Medical Education Annual Meeting in Daejeon, Korea.
Korean J Med Educ 2017 Sep; 29(3): 193-197.

https://doi.org/10.3946/kjme.2017.65

eISSN: 2005-7288

(C) The Korean Society of Medical Education. All rights reserved. This is an open-access article distributed under the terms of the Creative Commons Attribution Non-Commercial License (http:// creativecommons.org/licenses/by-nc/3.0/), which permits unrestricted non-commercial use, distribution, and reproduction in any medium, provided the original work is properly cited. 
domains that doctors need master in order to practice medicine. From the invention of the stethoscope and $\mathrm{X}$-ray machines, to the development of searchable electronic databases to the use of laser in surgery, technological advancements have been instrumental in improving the knowledge, diagnostic and management capability of doctors to care for patients. However, the knowledge domain is rapidly becoming a public domain with the general population having access to the same information that was once exclusive to doctors. While this changes the role of the doctor, giving patients access to an evidence-based e-health website can have positive repercussions by reducing doctor visits and empowering patients [2].

Diagnosis is a skill that takes doctors years if not decades to master and may increasingly be taken over by machines. Artificial intelligence and machines that are capable of deep learning hold significant promise. IBM's Watson has shown that machines can answer questions posed in natural language and respond with a precise accurate answer. Google's AlphaGo was able to use machine learning and neural networks to pick moves and win at a game previously thought to be too complex for a machine [3]. In medicine, deep learning computers have been able to identify malignant skin lesions as accurately as board-certified dermatologists [4]. Other technical and procedural skills may also become less important for doctors of the future when robotic surgeons become feasible, taking a lead from other industries where self-driving cars are in the pipeline and autopilot mode for flying commercial aircraft is already entrenched as usual practice. Even interpersonal and empathic skills may be given to machines as imagined in the 2013 science fiction move "Her" where a man develops a relationship with an intelligent computer operating system, and today where a humanoid robot named "Pepper" has been designed to welcome and take care of patients at a hospital in Belgium [5]. Automation and mechanization can bring much needed efficiency, accuracy, and better safety to health care and surprisingly, even a personal touch.

If knowledge and skills will not define the role of doctors in the future, then the focus for medical education will be on nurturing competency in a third domain. This could be considered the domain of "practical wisdom," the higher order ability to judge when and how to apply knowledge to best help individual patients in a compassionate way [6]. This may be learned through participation in frequent and diverse experiential learning opportunities, hands-on practice and discussion, self-awareness and reflection, and mentorship and role-modeling.

\section{Medical students: the Millennials}

The next generation of health care professionals will come from a cohort whose thinking and approach to life and career differ drastically from those who currently form the bulk of health care providers and educators. Medical educators will need to keep this in mind when designing and delivering curricula that will effectively enable these learners to flourish. These students of the Millennial Generation were born from 1981 to 2000 and are also known as "Digital Natives," "Generation Me," "Generation Y," or the "Net Generation." They tend to have certain general characteristics [7]: internalize that they are special, live sheltered lives, are self-confident (sometimes misguided thanks to helicopter parents), team-oriented, conventional (like to have everyone get along with each other), feel pressured (overprogrammed), and high-achieving (not necessarily realistic). Millennial learners therefore appreciate learning environments that allow them to multitask, use 
digital technology, share ideas, be interactive and experiential, work in teams, have flexibility and choice, and allow for personalization and mentorship [8].

\section{Medical education: adapting and in- novating -The University of Hong Kong as a case study}

The 6-year undergraduate bachelor of medicine/ bachelor of surgery (MBBS) curriculum at The University of Hong Kong is dynamic and ever-evolving to adapt to the needs of the population its graduates are poised to serve and the changing health care environment. Three initiatives are underway that aim to prepare graduates for the future by recognizing the gaps that technology cannot fill and tailoring the learning to the needs of the millennial learner.

\section{Bringing together the art and science of medicine: the Medical Humanities Pro- gramme}

Medical humanities are a means through which biomedically trained doctors, who will be working in difficult, uncertain, and emotionally charged conditions, can learn to better understand their patients and also learn to be more humanistic towards both their patients and themselves. The medical humanities engage the humanities, social sciences, and arts, which include literature, history, psychology, sociology, visual and dramatic arts, and music. By applying elements from these disciplines to medical education and medical practice, students learn about the human condition, develop skills of observation and critical thinking, and become more aware of the need for compassion and empathy.

This 6-year longitudinal programme was introduced to bring balance to a biomedically-centered curriculum.
Since 2012, all medical students explore the themes of "doctor and patient stories," "death, dying, and bereavement," "history of medicine," "culture, spirituality, and healing," and "humanitarianism and social justice" through narrative (reading literature, stories, and poetry), drama, visual arts and film, as well as through experiential (e.g., mindful practice, compassion workshop, and meeting carers of patients with mental illness) and service learning (elective opportunities locally and abroad). For example, in the first year, students build an awareness of their individual and collective self. By walking the path of the 1894 Hong Kong plague on the Tai Ping Shan Trail they consider the questions: Why do we practice medicine the way we do? What historical events in Hong Kong were critical in shaping our collective identity as doctors? By learning to meditate and to be in the moment, they explore the question: How does mindful practice help me develop the moment-to-moment non-judgemental awareness necessary to be fully present when doing my day-to-day activities and when I am seeing patients? By examining the perception of doctors held by the public, patients, and doctors themselves, through a filmmaker's lens, an artist's paintbrush, and a writer's pen, students begin to uncover clues to the existential question: Who am I?

\section{Pedagogical innovation: interprofessional team-based learning for health professions students}

Interprofessional education (IPE) involves students from two or more professions learning about, from and with each other to enable effective collaboration and improve health outcomes [9]. Team-based learning (TBL) is a structured format of learning that engages small groups to acquire and to apply the prerequisite knowledge to solve real-world problems. IPE and TBL were integrated into IPTBL (interprofessional team- 
based learning) in a deliberate effort to prepare students for the future reality of multidisciplinary team-based care. Since 2014, medical students have joined with students from 10 other health care disciplines to work on six instructional units ranging from "anticoagulation therapy" to "cancer" with face-to-face sessions taking place during one semester of their penultimate year of study. Groups of six to seven students are intentionally formed with representation from at least three different disciplines with 250-550 students in the teaching venue, depending on the instructional unit. Learning is dependent on teams sharing, deliberating, and coming to a consensus and is facilitated by a custom-made electronic platform as well as feedback and discussion with an interprofessional panel of teachers.

\section{Curriculum reform: an enrichment year}

A reconsideration of the MBBS curriculum resulted in an ambitious revamp which aims at enhancing and enriching students' total learning experience and whole person development by designating the entire year 3 as an enrichment year. Beginning in 2018, all students will select faculty-coordinated or self-initiated options from one of three thematic areas: research, service/humanitarian work, or intercalated degree/programme. These wholeyear learning opportunities will enable students to choose an area of interest in which to concentrate learning in order to fulfill designated criteria, engage substantively in the area of interest, and produce concrete deliverables upon completion of the year. An e-portfolio system to support a virtual community of learning will help students link their unique experiences with those of the other members of their small group, provide academic and social support, and remind students of the relevance of their collective experiences to the big picture of becoming a doctor.

\section{Conclusion}

The evolving medical landscape is rich with the promises of technology which has the potential to improve healthcare beyond our imagination. But we still need the human physician to do what machines cannot, to bring that third pillar of competency, practical wisdom, to the doctor-patient encounter. In the words of Professor Brian Hodges of the University of Toronto, we need the future doctor to "...integrate complex knowledge, adapt their expertise to different contexts, work in teams to address complex problems, and most importantly, find ways to convey a warm, compassionate and caring human presence [10]."

\section{ORCID:}

Julie Chen: https://orcid.org/0000-0002-7444-6182

Acknowledgements: None.

Funding: None.

Conflicts of interest: None.

\section{References}

1. Frenk J, Chen L, Bhutta ZA, et al. Health professionals for a new century: transforming education to strengthen health systems in an interdependent world. Lancet. 2010;376(9756):1923-1958.

2. Spoelman WA, Bonten TN, de Waal MW, et al. Effect of an evidence-based website on healthcare usage: an interrupted time-series study. BMJ Open. 2016;6(11): e013166.

3. Silver D, Huang A, Maddison CJ, et al. Mastering the game of Go with deep neural networks and tree search. Nature. 2016;529(7587):484-489. 
4. Esteva A, Kuprel B, Novoa RA, et al. Dermatologist-level classification of skin cancer with deep neural networks. Nature. 2017;542(7639):115-118.

5. Patients greeted by robot at Belgian hospital 2016. Reuters Web Site. http://in.reuters.com/article/us-techrobot-health-idINIKCNOZ30QJ. Accessed May 25, 2017.

6. Dowie A. Phronesis or "practical wisdom" in medical education. Med Teach. 2000;22(3):240-241.

7. Seven characteristics of Millennials. The Pennsylvania State University Web Site. https://sites.psu.edu/facultycenter/ 2012/01/21/strategies_for_teaching_millennial_students/. Accessed May 25, 2017.
8. Roberts DH, Newman LR, Schwartzstein RM. Twelve tips for facilitating Millennials' learning. Med Teach. 2012;34(4):274-278

9. Framework for action on interprofessional education and collaborative practice 2010. World Health Organization Web Site. http://www.who.int/hrh/resources/framework action/en/. Accessed May 25, 2017.

10. Brian Hodges: preparing the next generation 2015. Faculty of Medicine, University of Toronto Web Site. http://medicine.utoronto.ca/news/brian-hodges-preparingnext-generation. Accessed May 25, 2017. 